\title{
Anomalous Thermal Expansion of Cold-Rolled Ti-Nb-Ta-Zr Alloy
}

\author{
Masaaki Nakai ${ }^{1}$, Mitsuo Niinomi ${ }^{1}$, Toshikazu Akahori ${ }^{1}$, Harumi Tsutsumi ${ }^{1}$, \\ Xiaoliang Feng ${ }^{1, *}$ and Michiharu Ogawa ${ }^{2}$ \\ ${ }^{1}$ Institute for Materials Research, Tohoku University, Sendai 980-8577, Japan \\ ${ }^{2}$ Daido Steel Co., Ltd., Nagoya 457-8545, Japan
}

Negative thermal expansion, i.e. a type of shrinkage that occurs during heating, was observed in cold-rolled Ti-29Nb-13Ta-4.6Zr alloy (mass\%) (TNTZ). The reduction ratio of cold rolling and the angle of the longitudinal axis of specimens with respect to the cold-rolling direction were systematically changed, and then the thermal expansion rate was measured using a dilatometer. Further, the cyclicity of thermal expansion was examined for the cold-rolled TNTZ. From the results, it is observed that with an increase in the reduction ratio of cold rolling, the thermal expansion rate of TNTZ cold-rolled parallel to the rolling direction (RD) decreases, but it increases in TNTZ cold-rolled parallel to the transverse direction (TD). With regard to the anisotropy of thermal expansion, the thermal expansion rate increases with the angle between the longitudinal axis of the specimens and RD. Further, the cyclicity of the above-mentioned anomalous thermal expansion is observed in a temperature range below $473 \mathrm{~K}$, but it is not observed when the specimen is heated above $573 \mathrm{~K}$ in the first cycle. [doi:10.2320/matertrans.MRP2008380]

(Received October 14, 2008; Accepted November 25, 2008; Published January 15, 2009)

Keywords: titanium alloy, thermal expansion, cold rolling

\section{Introduction}

Thermal expansion is one of the intrinsic properties of any material that contains metals and alloys. Therefore, it is usually difficult to control their linear expansion coefficient. However, a multifunctional titanium alloy, its representative chemical composition being Ti-23Nb-0.7Ta-2Zr-O (at\%), has been developed by Saito et al.; it demonstrates Invar-like behavior, i.e. a quite low linear expansion coefficient, as one of the unique characteristics. ${ }^{1)}$ The Invar-like behavior is observed when this alloy is subjected to cold working. However, the cold-working conditions for inducing such a behavior have still not been clarified. On the other hand, Ti$29 \mathrm{Nb}-13 \mathrm{Ta}-4.6 \mathrm{Zr}$ alloy (mass\%) (TNTZ) developed by the authors $^{2,3)}$ has a similar chemical composition as that of the above-mentioned alloy. By analogy with chemical composition, the Invar-like behavior is likely to be observed in coldrolled TNTZ. Therefore, cold rolling was performed on TNTZ under various conditions, and its thermal expansion rate was measured systematically. Consequently, the coldrolling conditions under which the anomalous thermal expansion of the cold-rolled TNTZ occurred were clarified in this study.

\section{Experimental Procedures}

The material used in this study was a hot-forged bar of TNTZ (Ti: bal., Nb: 29.0, Ta: 13.2, Zr: 4.66, O: 0.12, N: 0.011 mass \%) with a diameter of $20 \mathrm{~mm}$. The bar was cut into cylindrical bulks with a thickness of $10 \mathrm{~mm}$. The bulks were solutionized at $1063 \mathrm{~K}$ for $3.6 \mathrm{ks}$ in vacuum, followed by icewater quenching $\left(\mathrm{TNTZ}_{\mathrm{ST}}\right)$. Next, $\mathrm{TNTZ}_{\mathrm{ST}}$ was cold-rolled into plates with reduction ratios of $10,30,50,70$, and $90 \%\left(\mathrm{TNTZ}_{\mathrm{CR} 10}, \mathrm{TNTZ}_{\mathrm{CR} 30}, \mathrm{TNTZ}_{\mathrm{CR} 50}, \mathrm{TNTZ}_{\mathrm{CR70}}\right.$, and $\mathrm{TNTZ}_{\mathrm{CR} 90}$, respectively) at room temperature in air, as shown in Fig. 1.

*Graduate student, Tohoku University

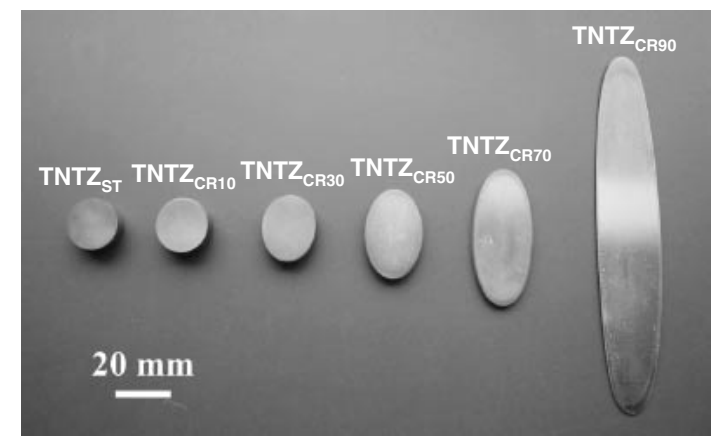

Fig. 1 Image of TNTZ after cold rolling at room temperature in air.

The constituted phases were then analyzed on the rolling plane by X-ray diffraction (XRD). The scanning angle range was $30-90^{\circ}$, and the scanning rate was $2^{\circ} \mathrm{min}^{-1}$. $\mathrm{Cu}-\mathrm{K} \alpha$ radiation was used at a voltage of $40 \mathrm{kV}$ and current of $150 \mathrm{~mA}$.

Specimens with a size of $1 \times 1 \times 12 \mathrm{~mm}^{3}$ were cut from the center part of cold-rolled plates for conducting a thermal expansion analysis; the longitudinal direction of cutting was systematically varied within the rolling plane of the coldrolled plates. The angle between the longitudinal axis of the specimens and the rolling direction (RD) was defined as $x$ and was set as 0 (RD), 30, 45, 60, and $90^{\circ}$ (transverse direction (TD)). The surface of the specimens was wet polished with emery papers of up to \#800 grit. In this experiment, two specimens cut from almost the same location were analyzed for repeatability. The thermal expansion analysis was conducted using a dilatometer in an argon atmosphere from 123 to $673 \mathrm{~K}$ at a heating rate of $10 \mathrm{~K} \mathrm{~min}^{-1}$.

\section{Experimental Results}

\subsection{Effect of cold rolling on microstructure of TNTZ}

Figure 2 presents the XRD profiles obtained from $\mathrm{TNTZ}_{\mathrm{ST}}$ and $\mathrm{TNTZ}_{\mathrm{CR} 10-90}$. These profiles are attributed to the planes 

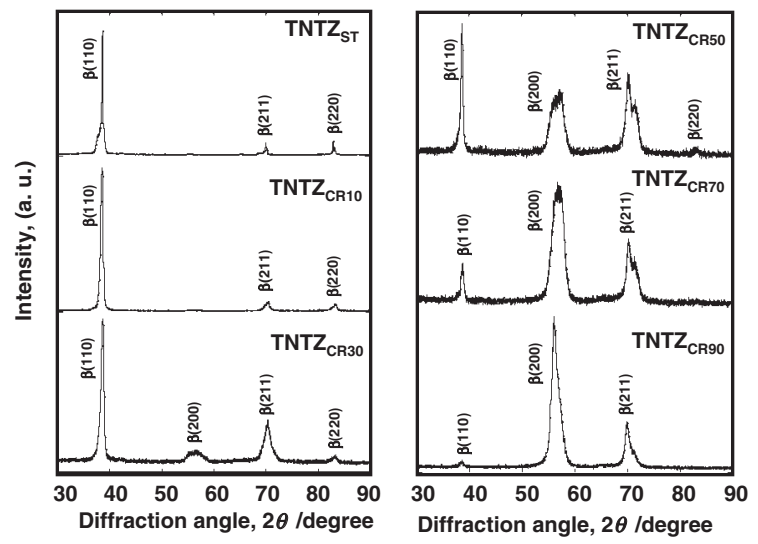

Fig. 2 XRD profiles obtained from rolling planes of $\mathrm{TNTZ}_{\mathrm{ST}}$ and $\mathrm{TNTZ}_{\mathrm{CR} 10-90}$.

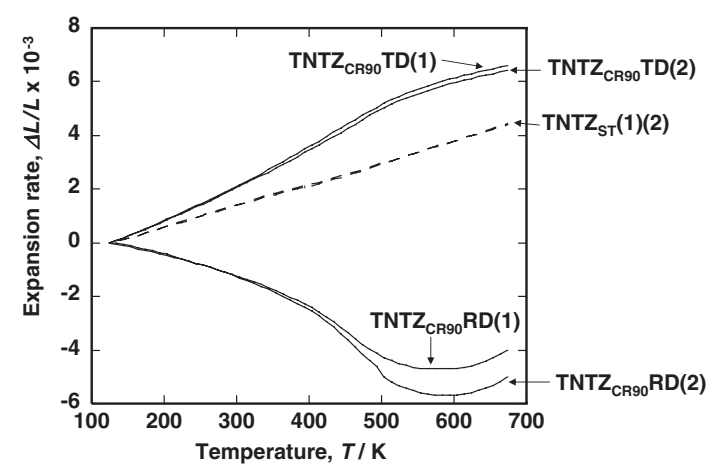

Fig. 3 Thermal expansion curves of $\mathrm{TNTZ}_{\mathrm{ST}}$ and $\mathrm{TNTZ}_{\mathrm{CR} 90}$ cold-rolled parallel to RD and TD.

parallel to the rolling plane of $\mathrm{TNTZ}_{\mathrm{CR} 10-90}$. As the reduction ratio increases, the peak intensity of $\beta(110)$ decreases, and that of $\beta(200)$ clearly increases. This result indicates that the texture is changed by cold rolling. On the other hand, according to our previous study, the stress-induced $\alpha^{\prime \prime}$ martensite transformation occurs in Ti-Nb-Ta- $\mathrm{Zr}$ alloys, and it is quite sensitive to $\mathrm{Nb}$ content. ${ }^{4)}$ Therefore, there is a possibility that the $\alpha^{\prime \prime}$ phase is induced in TNTZ by cold rolling. In this study, the peak of $\beta(200)$ is broadened and that of $\beta(211)$ is split; which may be caused by the stress-induced $\alpha^{\prime \prime}$ phase. However, the $\alpha^{\prime \prime}$ phase could not be identified clearly by XRD.

\subsection{Dependence of thermal expansion of TNTZ on direction of cold rolling}

Figure 3 illustrates the thermal expansion curves of TNTZ $_{\mathrm{ST}}$ and $\mathrm{TNTZ}_{\mathrm{CR} 90}$ cold-rolled parallel to the RD and TD. Normal thermal expansion is observed in $\mathrm{TNTZ}_{\mathrm{ST}}$; its thermal expansion rate increases with the temperature. However, in $\mathrm{TNTZ}_{\mathrm{CR} 90}$, thermal expansion is polarized; negative thermal expansion occurs during heating in TNTZ $_{\mathrm{CR} 90}$ cold-rolled parallel to the RD, but normal thermal expansion higher than that in $\mathrm{TNTZ}_{\mathrm{ST}}$ is observed in TNTZ $_{\mathrm{CR} 90}$ cold-rolled parallel to the TD. Figure 4 presents the thermal expansion curves of $\mathrm{TNTZ}_{\mathrm{ST}}$ and $\mathrm{TNTZ}_{\mathrm{CR} 90}$ for various values of $x\left(\mathrm{TNTZ}_{\mathrm{CR} 90}-x ; x=0,30,45,60\right.$, and $\left.90^{\circ}\right)$. Anisotropic thermal expansion is induced by cold rolling; the thermal expansion rate of TNTZ $\mathrm{CR}_{\mathrm{C} 90}$ increases with $x$.

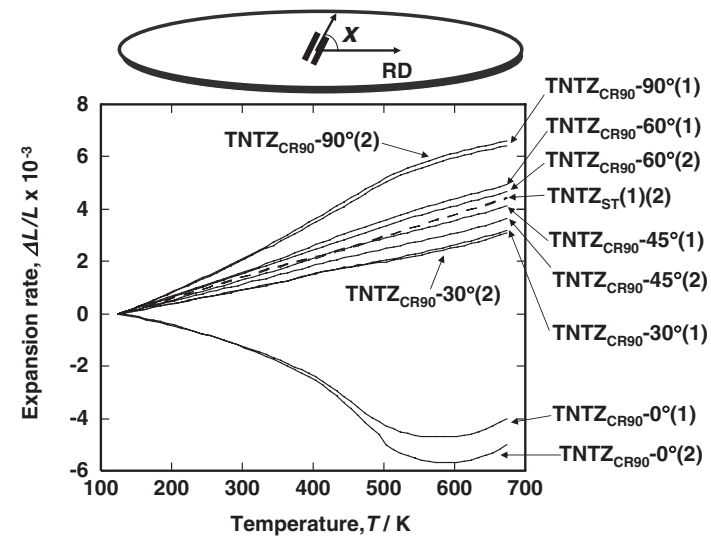

Fig. 4 Thermal expansion curves of TNTZ $\mathrm{TT}_{\mathrm{ST}}$ and $\mathrm{TNTZ}_{\mathrm{CR} 90}$ for $x=0,30$, 45,60 , and $90^{\circ}$, which are angles between longitudinal axis of specimen and RD.

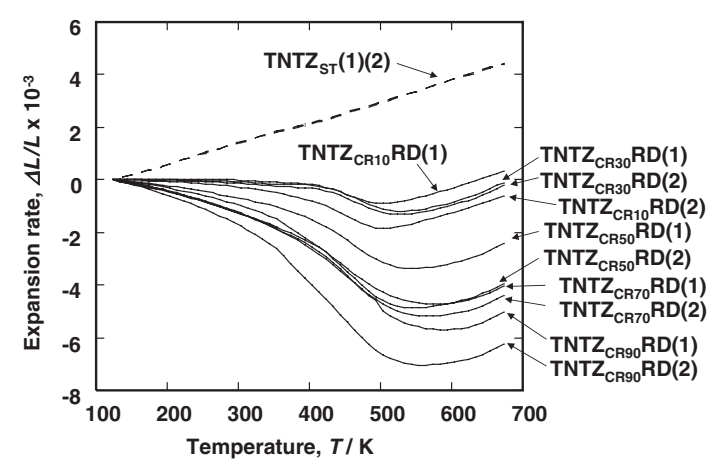

Fig. 5 Thermal expansion curves of $\mathrm{TNTZ}_{\mathrm{ST}}$ and $\mathrm{TNTZ}_{\mathrm{CR} 10-90}$ cold-rolled parallel to RD.

\subsection{Dependence of thermal expansion of TNTZ on reduction ratio of cold rolling}

Figure 5 presents the thermal expansion curves of TNTZ $_{\mathrm{ST}}$ and $\mathrm{TNTZ}_{\mathrm{CR} 10-90}$ cold-rolled parallel to the RD. As the reduction ratio of cold rolling increases, the thermal expansion rate decreases; the Invar-like behavior in a temperature range from $100 \mathrm{~K}$ to $400 \mathrm{~K}$ and negative thermal expansion during heating in a temperature range from $100 \mathrm{~K}$ to $500 \mathrm{~K}$ are observed in $\mathrm{TNTZ}_{\mathrm{CR} 10-30}$ and $\mathrm{TNTZ}_{\mathrm{CR} 50-90}$, respectively. Figure 6 illustrates the thermal expansion curves of $\mathrm{TNTZ}_{\mathrm{ST}}$ and $\mathrm{TNTZ}_{\mathrm{CR} 10-90}$ cold-rolled parallel to the TD. Normal thermal expansion is observed in every TNTZ specimen. However, the thermal expansion rate of $\mathrm{TNTZ}_{\mathrm{CR} 10-30}$ is lower than that of $\mathrm{TNTZ}_{\mathrm{ST}}$. In contrast, higher thermal expansion is observed in $\mathrm{TNTZ}_{\mathrm{CR} 50-90}$ than in TNTZ $_{\mathrm{ST}}$.

\subsection{Cyclicity of thermal expansion of cold-rolled TNTZ}

Figure 7 indicates the cyclic thermal expansion of TNTZ $_{\text {CR50 }}$ cold-rolled parallel to the RD. After heating in the temperature range below $473 \mathrm{~K}$ in the first cycle, negative thermal expansion is maintained during heating even after the second cycle, as shown in Figs. 7(a) and (b). However, when the specimen is heated above $573 \mathrm{~K}$ in the first cycle, negative thermal expansion does not occur during heating, and normal thermal expansion occurs after the second cycle, as shown in Figs. 7(c) and (d). 


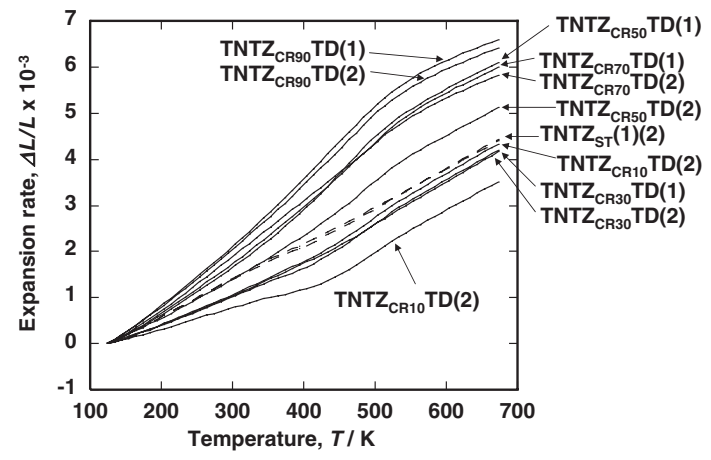

Fig. 6 Thermal expansion curves of $\mathrm{TNTZ}_{\mathrm{ST}}$ and $\mathrm{TNTZ}_{\mathrm{CR} 10-90}$ cold-rolled parallel to TD.

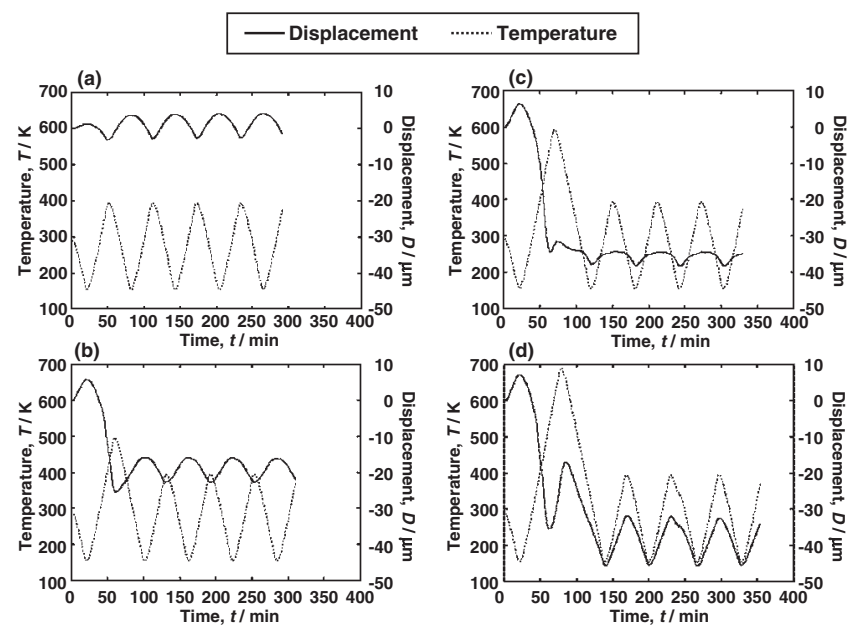

Fig. 7 Cyclic thermal expansion of $\mathrm{TNTZ}_{\mathrm{CR} 50}$ heated up to (a) $373 \mathrm{~K}$, (b) $473 \mathrm{~K}$, (c) $573 \mathrm{~K}$, and (d) $673 \mathrm{~K}$ in first cycle and then up to $373 \mathrm{~K}$ after second cycle.

\section{Discussion}

With regard to finding the cause for the Invar-like behavior, except that for the conventional magnetic effect, ${ }^{5)}$ a martensitic transformation behavior of $\mathrm{Cu}$-based shape memory alloys was investigated to study the change in their thermal expansion rate. ${ }^{6,7)}$ Studies regarding the shape memory effect and superelasticity of Ti-Nb-based alloys that demonstrate $\beta$-phase stability similar to TNTZ have been reported in large numbers. ${ }^{8-11)}$ These properties are a result of heat- and stress-induced $\alpha^{\prime \prime}$ martensitic transformations and their reversions. Therefore, it is highly possible that the anomalous thermal expansion of cold-rolled TNTZ may be related to the heat- and stress-induced $\alpha^{\prime \prime}$ martensitic transformations and their reversions.

On the other hand, the shape memory and superelastic performances of titanium alloys are affected by their texture because there exists a specific lattice correspondence between $\alpha^{\prime \prime}$ and $\beta$ phases as shown in Fig. 8. ${ }^{8,9,12,13)}$ $\alpha^{\prime \prime}[100], \alpha^{\prime \prime}[010]$, and $\alpha^{\prime \prime}[001]$ are parallel to $\beta[100]$, $\beta[011]$, and $\beta[0 \overline{1} 1]$, respectively. According to the lattice constants of $\alpha^{\prime \prime}$ phase $\left(a_{\alpha^{\prime \prime}}, b_{\alpha^{\prime \prime}}\right.$, and $\left.c_{\alpha^{\prime \prime}}\right)$ and $\beta$ phase $\left(a_{\beta}\right)$, $\sqrt{2} a_{\beta}$ is smaller than $b_{\alpha^{\prime \prime}}$, but similar to $c_{\alpha^{\prime \prime}}$ in Ti-Nb-based alloys. ${ }^{8,9,13,14)}$ Depending on chemical composition, $c_{\alpha^{\prime \prime}}$ is slightly smaller ${ }^{8,9,13)}$ or larger ${ }^{9,14)}$ than $\sqrt{2} a_{\beta}$. Further, $a_{\beta}$ is

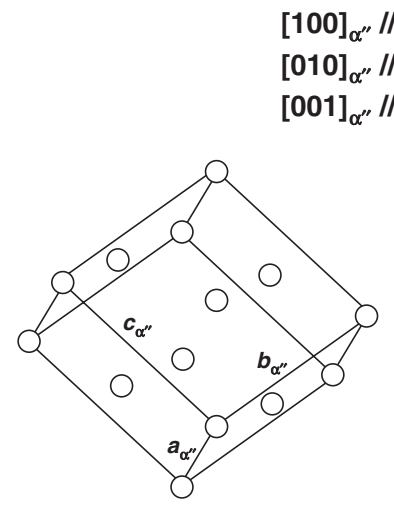

(a) $\alpha^{\prime \prime}$ phase $[100]_{\alpha^{\prime \prime}} / /[100]_{\beta}$

$[010]_{\alpha^{\prime \prime}} / /[011]_{\beta}$

$001]_{\alpha^{\prime \prime}} / /[011]_{\beta}$

Fig. 8 Lattice correspondence between (a) $\alpha^{\prime \prime}$ and (b) $\beta$ phases.

larger than $a_{\alpha^{\prime \prime}}$ in them. On the other hand, the accumulation of $\beta(110)$ is usually observed on the cross section perpendicular to RD in $\beta$-type titanium alloys. ${ }^{14)}$ In this study, the texture of TNTZ was changed by cold rolling, as shown in Fig. 2. Further, the opposite expansion behaviors-negative and normal thermal expansions-were observed in the specimens cut parallel to the RD and TD from the same cold-rolled plate, as shown in Fig. 3. If it is assumed that RD and TD is parallel to $\beta[011]$ and $\beta[0 \overline{1} 1]$ respectively, these behaviors can be explained by the martensitic transformation from $\alpha^{\prime \prime}$ phase to $\beta$ phase; the decrease in the length from $b_{\alpha^{\prime \prime}}$ to $\sqrt{2} a_{\beta}$ corresponds to the negative thermal expansion of TNTZ cold-rolled parallel to the RD. With regard to TD, thermal expansion behavior may be dependent on length ratio of $c_{\alpha^{\prime \prime}}$ to $\sqrt{2} a_{\beta}$. If $c_{\alpha^{\prime \prime}}$ is smaller than $\sqrt{2} a_{\beta}$, the normal thermal expansion, whose rate is higher than $\mathrm{TNTZ}_{\mathrm{ST}}$, is observed for TNTZ cold-rolled parallel to the TD.

As mentioned above, the martensitic transformation and texture are possibly the key factors affecting the anomalous thermal expansion of cold-rolled TNTZ. However, the obvious experimental evidence is still not obtained in this study. The mechanism will be elucidated more clearly by the further crystallographic experiments in the future study.

\section{Conclusions}

In this study, the thermal expansion of cold-rolled Ti$29 \mathrm{Nb}-13 \mathrm{Ta}-4.6 \mathrm{Zr}$ alloy (TNTZ) was systematically examined by varying the cold-rolling conditions such as by varying the reduction ratio from 0 to $90 \%$ and varying the angle of the longitudinal axis of the specimens with respect to the cold-rolling direction from 0 to $90^{\circ}$. Further, cyclicity of thermal expansion was also examined for the cold-rolled TNTZ. The following results were obtained:

(1) The XRD profiles obtained from the rolling planes of TNTZ show that the peak intensity of $\beta(110)$ decreases, and that of $\beta(200)$ increases with the reduction ratio of cold rolling.

(2) For the cold-rolled TNTZ with the reduction ratio of $90 \%\left(\mathrm{TNTZ}_{\mathrm{CR} 90}\right)$, thermal expansion is polarized; negative thermal expansion occurs during heating in $\mathrm{TNTZ}_{\mathrm{CR} 90}$ cold-rolled parallel to the rolling direction (RD), and normal thermal expansion occurs in 
TNTZ $_{\mathrm{CR} 90}$ cold-rolled parallel to the transverse direction (TD).

(3) With an increase in the reduction ratio of cold rolling, the thermal expansion rate decreases in TNTZ coldrolled parallel to the RD but increases in TNTZ coldrolled parallel to the TD. The Invar-like behavior and negative thermal expansion during heating are observed in $\mathrm{TNTZ}_{\mathrm{CR} 10-30}$ and $\mathrm{TNTZ}_{\mathrm{CR} 50-90}$, respectively.

(4) Anisotropic thermal expansion is observed in $\mathrm{TNTZ}_{\mathrm{CR} 90}$. The thermal expansion rate increases with the angle between the longitudinal axis of specimen and the RD, which is set as $0,30,45,60$, and $90^{\circ}$.

(5) Cyclic negative thermal expansion is observed during heating in $\mathrm{TNTZ}_{\mathrm{CR} 50}$ after the second cycle when the specimen is heated in the temperature range below $473 \mathrm{~K}$ in the first cycle. However, when the specimen is heated to above $573 \mathrm{~K}$ in the first cycle, the anomalous behavior is not observed after the second cycle.

\section{Acknowledgment}

The authors would like to express their thanks to Mr. N. Harima, IMR, Tohoku University (Sendai, Japan), for the useful discussion about plastic deformation. This work was supported in part by the Global COE Program "Materials Integration International Center of Education and Research, Tohoku University", Ministry of Education, Culture, Sports, Science and Technology (MEXT) (Tokyo, Japan), and the cooperative research program of the Advanced Research Center of Metallic Glasses, Institute for Materials Research, Tohoku University (Sendai, Japan).

\section{REFERENCES}

1) T. Saito et al.: Science 300 (2003) 464-467.

2) D. Kuroda, M. Niinomi, M. Morinaga, Y. Kato and T. Yashiro: Mater. Sci. Eng. A 243 (1998) 244-249.

3) M. Niinomi: Biomater. 24 (2003) 2673-2683.

4) M. Niinomi, T. Akahori, M. Nakai and N. Kawakita: Proc. Materials and Processes for Medical Devices (MPMD) 2007 Conference \& Exposition, (ASM Int., 2007), in press.

5) R. J. Weiss: Proc. Phys. Soc. London 82 (1963) 281-288.

6) R. Kainuma, J. J. Wang, T. Omori, Y. Sutou and K. Ishida: Appl. Phys. Lett. 80 (2002) 4348-4350.

7) J. J. Wang, T. Omori, Y. Sutou, R. Kainuma and K. Ishida: J. Eectron. Mater. 33 (2004) 1098-1102.

8) H. Y. Kim, T. Sasaki, K. Okutsu, J. I. Kim, T. Inamura, H. Hosoda and S. Miyazaki: Acta Mater. 54 (2006) 423-433.

9) H. Y. Kim, Y. Ikehara, J. I. Kim, H. Hosoda and S. Miyazaki: Acta Mater. 54 (2006) 2419-2429.

10) J. I. Kim, H. Y. Kim, T. Inamura, H. Hosoda and S. Miyazaki: Mater. Sci. Eng. A 403 (2005) 334-339.

11) S. Miyazaki, H. Y. Kima and H. Hosoda: Mater. Sci. Eng. A 438-440 (2006) 18-24

12) D. S. Lieberman, M. S. Wechsler and T. A. Read: J. Appl. Phys. 26 (1955) 473-484

13) T. Inamura, Y. Fukui, H. Hosoda, K. Wakashima and S. Miyazaki: Mater. Trans. 45 (2004) 1083-1089.

14) H. Matsumoto, S. Watanabe and S. Hanada: J. Alloy. Compd. 439 (2007) 146-155. 